\title{
Twist promotes invasion and cisplatin resistance in pancreatic cancer cells through growth differentiation factor $\mathbf{1 5}$
}

\author{
HONG JI, HONG-WEI LU, YI-MING LI, LE LU, JIN-LONG WANG, YA-FEI ZHANG and HAO SHANG \\ Department of General Surgery, The Second Affiliated Hospital, Xi'an Jiaotong University, \\ Xi'an, Shaanxi 710004, P.R. China
}

Received August 17, 2014; Accepted May 6, 2015

DOI: $10.3892 / \mathrm{mmr} .2015 .3867$

\begin{abstract}
Pancreatic cancer (PC) is an aggressive and devastating disease with a poor prognosis. Cisplatin, a commonly used chemotherapeutic agent for solid tumors, is effective as a single agent or in combination with other drugs for the treatment of PC. Previous studies have suggested that Twist and growth differentiation factor 15 (GDF15) are involved in the progression of PC. However, the role of Twist and GDF15 in PC remains to be elucidated. In the present study, the individual effect of and interaction between Twist and GDF15 in PC cell invasion and chemoresistance to cisplatin was examined. Twist and/or GDF15 were stably overexpressed or knocked down in ASPC-1 and BXPC-3 human PC cells. Overexpression of Twist in the two cell lines markedly increased GDF15 expression, cell invasion, matrix metalloproteinase- 2 expression/activity and the half maximal inhibitory concentration (IC50) values of cisplatin, which was eradicated by GDF15 knockdown or the selective p38 mitogen-activated protein kinase (MAPK) inhibitor SB203580 $(10 \mu \mathrm{M})$. By contrast, Twist knockdown significantly decreased GDF15 expression, cell invasion, matrix metalloproteinase- 2 expression/activity and the IC50 values of cisplatin, which was completely reversed by overexpression of GDF15. In addition, while overexpression and knockdown of Twist increased and decreased p38 MAPK activity, respectively, GDF15 demonstrated no significant effect on p38 MAPK activity in PC cells. In conclusion, the present study, for the first time, to the best of our knowledge, demonstrated that Twist promotes PC cell invasion and cisplatin chemoresistance through inducing GDF15 expression via a p38 MAPK-dependent mechanism. The present study provides new insights into the molecular mechanisms underlying PC progression and chemoresistance.
\end{abstract}

Correspondence to: Dr Hong-Wei Lu, Department of General Surgery, The Second Affiliated Hospital, Xi'an Jiaotong University, 157 West Road, Xi'an, Shaanxi 710004, P.R. China

E-mail:1hwdoc@163.com

Key words: twist, growth differentiation factor 15, pancreatic cancer, invasion, chemoresistance, cisplatin, p38 protein-activated protein kinase, matrix metalloproteinase-2

\section{Introduction}

Pancreatic cancer (PC) is an aggressive and devastating disease with a poor prognosis (1). It is the eighth most common cause of cancer-associated mortality in the world $(2,3)$ and leads to 227,000 mortalities worldwide every year. The 5-year survival rate in patients with $\mathrm{PC}$ is $<5 \%$ (4). Cisplatin, a commonly used chemotherapeutic agent for solid tumors (5), is effective as a single agent or in combination with other drugs for the treatment of PC $(6,7)$.

Twist, also known as Twist1, belongs to the basic helix-loop-helix transcription factor family. A high expression of Twist has been detected in several types of cancer and has been associated with the initial phase of metastatic progression (8). A previous study demonstrated that Twist is upregulated in PC tissues, suggesting that Twist is involved in the progression of PC (9).

Growth differentiation factor 15 (GDF15), also termed macrophage inhibitory cytokine-1, is a divergent member of the transforming growth factor- $\beta$ superfamily. It has multiple roles in various pathologies, including inflammation, cancer, cardiovascular diseases and obesity $(10,11)$. In cancer, GDF15 has been reported to have tumorigenic and anti-tumorigenic activities $(11,12)$. Although the role of GDF15 in tumorigenesis is most likely not universal in all types of cancer, it is elevated in the serum of PC patients compared with healthy controls and those with benign pancreatic neoplasms $(13,14)$. A previous study has demonstrated that serum GDF15 could be used as a diagnostic biomarker with high sensitivity and specificity for identifying PC (15).

These previous studies suggest that Twist and GDF15 are involved in PC progression. However, the roles of Twist and GDF15 in PC remain to be elucidated. The present study examined the interaction between Twist and GDF15 in PC cell invasion and chemoresistance to cisplatin.

\section{Materials and methods}

Cell lines, plasmids and reagents. The human $\mathrm{PC}$ cell lines ASPC-1 (CRL-1682) and BXPC-3 (CRL-1687) were purchased from the American Type Culture Collection (ATCC, Manassas, VA, USA). Twist (sc-38604-V) and GDF15 (sc-39798-V) shRNA lentiviral particles, control shRNA lentiviral particles-A (cat. no. sc-108080) and mouse anti-human 
monoclonal Twist antibody (Twist2C1a; cat. no. sc-81417), mouse anti-human GDF-15 monoclonal antibody (G-5; cat. no. sc-377195) and mouse anti-human matrix metalloproteinase-2 monoclonal antibody (MMP-2; cat. no. sc-53630) were purchased from Santa Cruz Biotechnology, Inc. (Santa Cruz, CA, USA). The SensoLyte 520 MMP-2 Assay kit (cat. no. AS-71151) was purchased from AnaSpec (Fremont, CA, USA). The QCM ECMatrix 24-well ( $8 \mu \mathrm{M})$ Fluorimetric Cell Invasion Assay kit (cat. no. ECM554) was purchased from Chemicon (Millipore, Billerica, MA, USA). Superfect transfection reagent was purchased from Qiagen (Valencia, CA, USA). The p38 mitogen-activated protein kinase (MAPK) Assay kit (cat. no. 9820) was purchased from Cell Signaling Technology, Inc. (Beverly, MA, USA). Human Twist cDNA was subcloned into a pcDNA 3.1 expression vector (16). Full-length human GDF15 cDNA (MCG: 4145) vector was purchased from Invitrogen Life Technologies (Carlsbad, CA, USA). The human GDF15 expression vector (pcDNA3-GDF15) was constructed by subcloning the GDF15 cDNA vector following digestion with EcoRI and NotI into the pcDNA3.1 expression vector (Invitrogen Life Technologies). Puromycin, G418, cisplatin, the selective p38 MAPK inhibitor SB203580 and all chemicals of reagent grade were purchased from Sigma-Aldrich (St. Louis, MO, USA).

Transfection and lentiviral transduction. The Twist or GDF15 expression vector was transfected into cells using Superfect transfection reagent (Qiagen) according to the manufacturer's instructions. Pools of stable transductants were generated via selection with G418 $(600 \mu \mathrm{g} / \mathrm{ml})$ according to the manufacturer's instructions. The Twist or GDF15 shRNA lentiviral particles contain expression constructs encoding target-specific 19-25 nt (plus hairpin) shRNA designed to specifically knockdown Twist or GDF15 gene expression. The control shRNA lentiviral particles contain a scrambled shRNA sequence that does not lead to specific degradation of any cellular mRNA and was used as a negative control. Lentiviral transduction was performed in ASPC-1 and BXPC-3 cells. Pools of stable transductants were generated via selection with puromycin (4 $\mu \mathrm{g} / \mathrm{ml}$ ) according to the manufacturer's instructions (Santa Cruz Biotechnology, Inc.).

Reverse transcription quantitative polymerase chain reaction $(R T-q P C R)$. RNA was prepared from cells using TRIzol reagent followed by purification using a TURBO DNA-free kit (Ambion, Austin, TX, USA). The cDNAs were synthesized using SuperScript II reverse transcriptase (Invitrogen Life Technologies). qPCR was performed on the LightCycler thermal cycler system (Roche Diagnostics, Indianapolis, IN, USA) using an SYBR Green I kit (Roche Diagnostics) according to the manufacturer's instructions. The results were normalized against that of the housekeeping gene glyceraldehyde-3-phosphate dehydrogenase (GAPDH) in the same sample. The primers used are as follows: Human GDF15, forward 5'-CGGTGAATGGCTCTCAGATG-3' and reverse 5'-CAGGTCCTCGTAGCGTTTCC-3'; human GAPDH, forward 5'-GACTCATGACCACAGTCCATGC-3' and reverse 5'-AGAGGCAGGGATGATGTTCTG-3'. Each experiment was repeated three times in duplicate.
Cell invasion assay. In vitro cell invasion assays were performed with the QCM ECMatrix 24-well $(8 \mu \mathrm{M})$ Fluorimetric Cell Invasion Assay kit (Chemicon; Millipore) according to the manufacturer's instructions $(17,18)$. The kit used an insert polycarbonate membrane with an $8 \mu \mathrm{M}$ pore size. The insert in the invasion kit was coated with a thin layer of ECMatrix. Cell invasion was determined by fluorescence. Each experiment was repeated three times in duplicate.

Western blot analysis. Cells were dissolved in $250 \mu 1$ of $1 \mathrm{X}$ SDS loading buffer (62.5 mm TrisHCl, pH 6.8, 2\% SDS, 25\% glycerol, $0.01 \%$ bromphenol blue, $5 \%$ 2-mercaptoethanol) and incubated at $95^{\circ} \mathrm{C}$ for $10 \mathrm{~min}$. Equal quantities of proteins for each sample were separated by $10 \%$ SDS-polyacrylamide gel and blotted onto a polyvinylidene difluoride microporous membrane (Millipore). Membranes were incubated for $1 \mathrm{~h}$ at room temperature with a 1:500 dilution of the following primary antibodies: Anti-Twist, anti-GDF-15, and anti-MMP-2, and then washed and revealed using horseradish peroxidase-conjugated bovine anti-mouse secondary antibodies (cat. no. sc-2371; Santa Cruz Biotechnology, Inc., 1:5,000, $1 \mathrm{~h}$ ). Peroxidase was revealed with a GE Healthcare ECL kit (Shanghai, China). Three independent experiments were performed for each western blot analysis.

MMP-2 activity assay. MMP-2 activity was measured with the SensoLyte 520 MMP-2 Assay kit (AnaSpec) according to the manufacturer's instructions $(19,20)$. The supernatants were collected and then incubated with 4-aminophenylmercuric acetate and MMP-2 substrate. The fluorescence intensity at Ex/Em wavelengths of $490 \mathrm{~nm} / 520 \mathrm{~nm}$ were used as a measure of MMP-2 activity. Each experiment was repeated three times in duplicate.

Cisplatin chemosensitivity assay. Cells were plated in triplicate in 96-well plates at a density of 5,000 cells. After $24 \mathrm{~h}$ of incubation, the medium was replaced by fresh medium with or without various concentrations of cisplatin $(0.1,0.25,0.5$, 1.0, 1.5, 3.0, 6.0, 15.0, 30.0, or 55.0 mM) (Sigma-Aldrich). Subsequently, cell viability was assayed $48 \mathrm{~h}$ later using a modified MTT assay as previously described (21). The half maximal inhibitory concentration (IC50) values were defined as the concentrations resulting in a $50 \%$ reduction in growth compared with control cell growth.

p38 MAPK activity assay. p38 MAPK activity was measured using the p38 MAPK Assay kit (Cell Signaling Technology, Inc.) according to the manufacturer's instructions (22). Briefly, cells were directly lysed in the culture dishes. Cell lysates were sonicated and centrifuged at $20,000 \mathrm{x}$ g for $10 \mathrm{~min}$ at $4^{\circ} \mathrm{C}$. The supernatant containing equivalent quantities of protein (200 $\mu \mathrm{g}$ ) was incubated by gentle rocking with $20 \mu \mathrm{l}$ of immobilized mouse anti-human phospho-p38-MAPK monoclonal antibody (28B10; cat. no. 9216; Cell Signaling Technology, Inc.; 1:500) for $16 \mathrm{~h}$ at $4^{\circ} \mathrm{C}$. The immunoprecipitates were washed twice with the lysing buffer and pelleted by centrifugation at 20,000 x g for $10 \mathrm{~min}$ at $4^{\circ} \mathrm{C}$. The p38 MAPK assay was performed using activating transcription factor 2 (ATF2) fusion protein $(2 \mu \mathrm{g})$ as a substrate in the presence of $200 \mu \mathrm{M}$ ATP and $1 \mathrm{X}$ kinase buffer according to the manufacturer's 
A
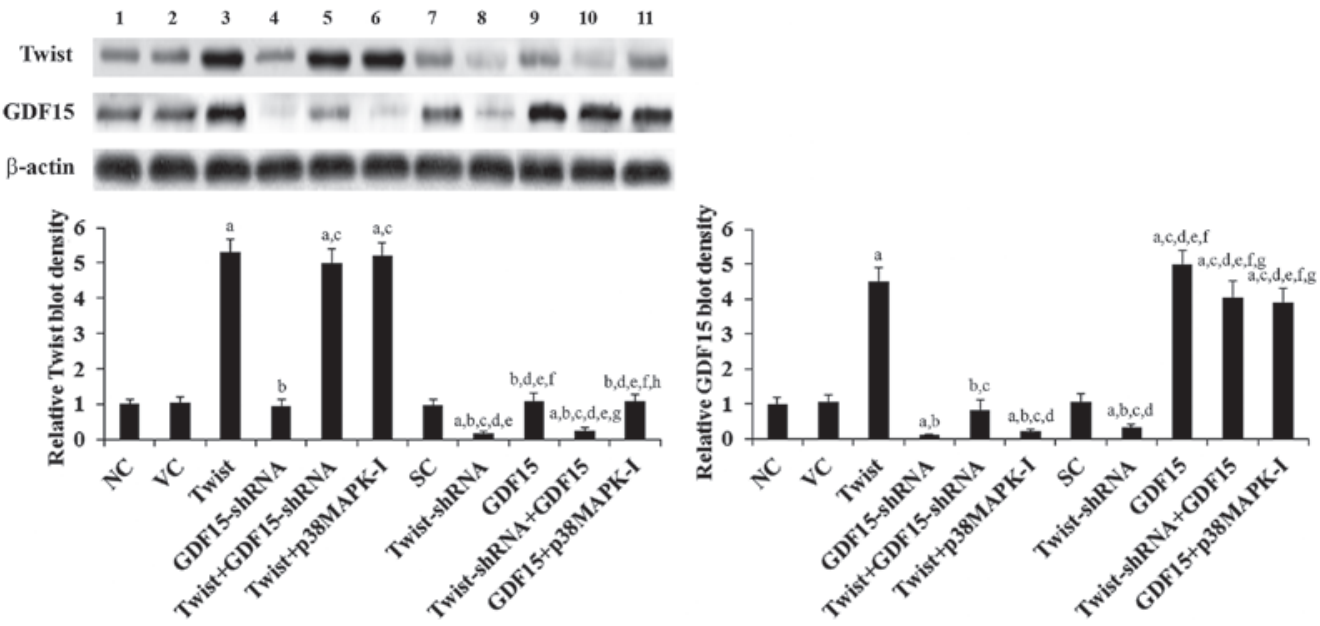

B
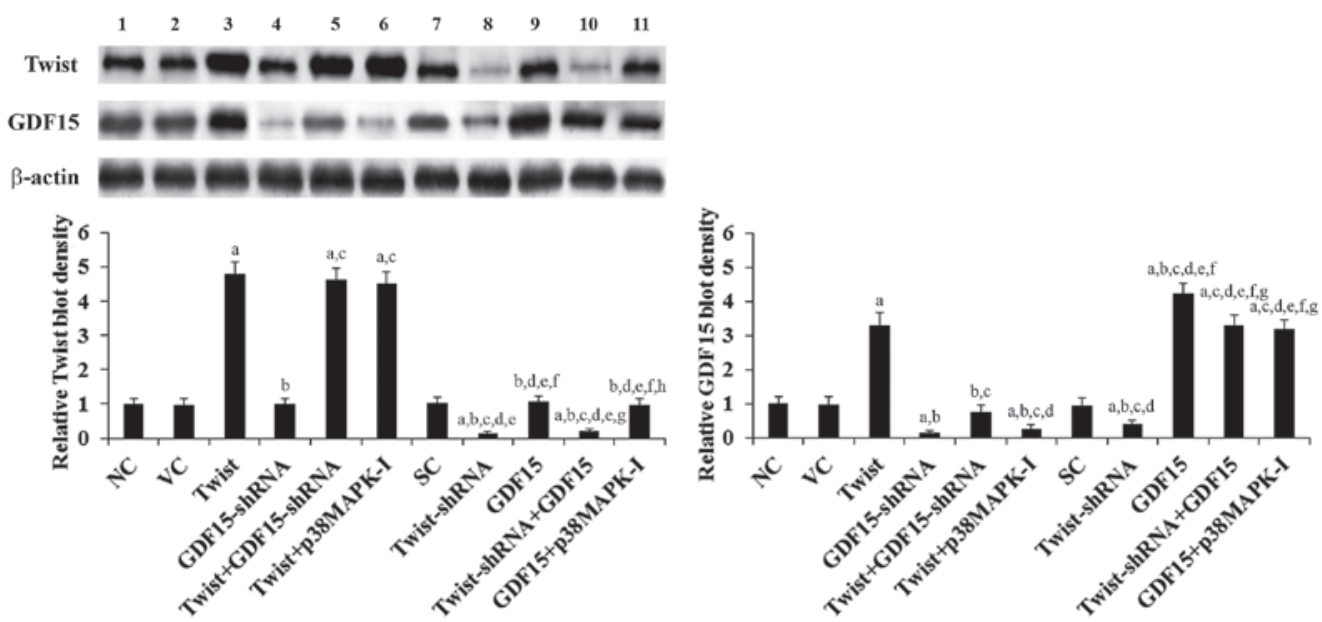

Figure 1. Protein levels of Twist and GDF15 in human PC cells. In (A) ASPC-1 and (B) BXPC-3 human PC cells, the protein levels of Twist and GDF15 were determined using western blot analysis in $\mathrm{NC}$ cells (NC, lane 1), cells stably transfected with the empty pcDNA3.1 vector (VC, lane 2), cells stably transfected with the pcDNA3-Twist expression vector (Twist, lane 3), cells stably transduced with GDF15-shRNA (lane 4), cells stably transfected with Twist and transduced with GDF15-shRNA (Twist + GDF15-shRNA, lane 5), cells stably transfected with Twist and treated with the selective p38 MAPK inhibitor SB203580 $(10 \mu \mathrm{M})$ for $30 \mathrm{~min}$ (Twist + p38MAPK-I, lane 6), cells stably transduced with SC shRNA (SC, lane 7), cells stably transduced with Twist-shRNA (lane 8), cells stably transfected with the pcDNA3-GDF15 expression vector (GDF15, lane 9), cells stably transduced with Twist-shRNA and transfected with GDF15 (Twist-shRNA + GDF15, lane 10) and cells stably transfected with GDF15 and treated with SB203580 (10 $\mu$ M) for 30 min (GDF15 + p38MAPK-I, lane 11). $\beta$-actin was used as a loading control. The density of Twist and the GDF15 blots was normalized against that of the $\beta$-actin blot to obtain a relative blot density, which is expressed as fold changes to that of $\mathrm{NC}$ (designated as 1). Three independent experiments were performed for each western blot analysis. Data values are expressed as the mean + standard deviation. ${ }^{a} \mathrm{P}<0.05$ vs. controls ( $\mathrm{NC}, \mathrm{VC}$ and $\mathrm{SC}$ ); ${ }^{\mathrm{b}} \mathrm{P}<0.05$ vs. Twist; ${ }^{\mathrm{c}} \mathrm{P}<0.05$ vs. GDF 15 -shRNA; ${ }^{\mathrm{d}} \mathrm{P}<0.05$ vs. Twist + GDF15-shRNA; ${ }^{\mathrm{e}}<<0.05$ vs. Twist + p38MAPK-I; ${ }^{\mathrm{f}} \mathrm{P}<0.05$ vs. Twist-shRNA; ${ }^{\mathrm{g}} \mathrm{P}<0.05$ vs. GDF15; ${ }^{\mathrm{h}} \mathrm{P}<0.05$ vs. Twist-shRNA + GDF15. GD15, growth differentiation factor 15; PC, pancreatic cancer; NC, normal control; SC, scrambled control; MAPK, mitogen-activated protein kinase.

instructions. Samples were resolved on a $12 \%$ SDS-PAGE gel and visualized by autoradiography.

Statistical analysis. Statistical analyses were performed with SPSS for Windows 19.0 (SPSS, Inc., Chicago, IL, USA). All data values are expressed as the mean \pm standard deviation. Comparisons of means among multiple groups were performed with one-way analysis of variance followed by post hoc pairwise comparisons using Tukey's test. $\mathrm{P}<0.05$ was considered to indicate a statistically significant difference.

\section{Results}

Overexpression and knockdown of Twist and GDF15 in human PC cells. To investigate the functional interaction between Twist and GDF15 in PC cells, Twist and GDF15 were stably overexpressed in ASPC-1 and BXPC-3 human PC cells by stable transfection. By contrast, the cells were also stably transduced with lentiviral shRNAs to knock down Twist and GDF15, respectively. As shown in Fig. 1, Twist and GDF15 were constitutively expressed in ASPC-1 and BXPC-3 cells. Compared with the controls, Twist was overexpressed $>4.8$ fold and knocked down $>80 \%$ in ASPC-1 and BXPC-3 cells, respectively; GDF was overexpressed $>4.2$ fold and knocked down $>80 \%$ in ASPC-1 and BXPC-3 cells, respectively. GDF15 expression in the cells increased (by $>3.3$ fold) and decreased $(>60 \%)$ in parallel with Twist overexpression and knockdown, respectively. By contrast, overexpression and knockdown of GDF15 had no significant effect on Twist expression (Fig. 1). Our pilot study suggested that Twist regulates GDF15 

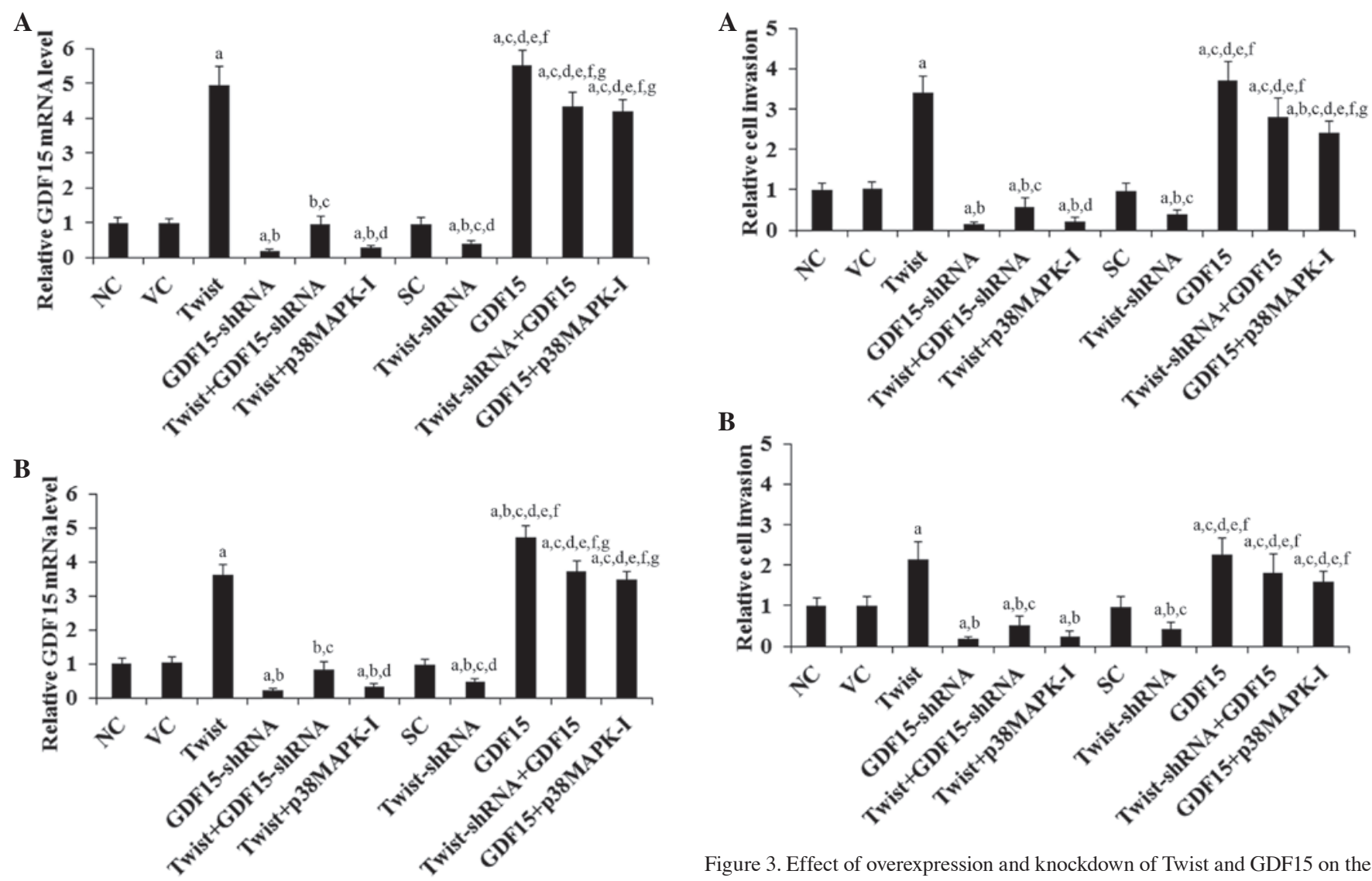

Figure 2. mRNA levels of Twist and GDF15 in PC cells. In (A) ASPC-1 and (B) BXPC-3 PC cells, the mRNA levels of Twist and GDF15 were determined by reverse transcription quantitative polymerase chain reaction in NC cells, cells stably transfected with the empty pcDNA3.1 vector (VC), cells stably transfected with Twist, cells stably transduced with GDF15-shRNA, cells stably transfected with Twist and transduced with GDF15-shRNA (Twist + GDF15-shRNA), cells stably transfected with Twist and treated with the selective p38 MAPK inhibitor SB203580 $(10 \mu \mathrm{M})$ for 30 min (Twist + p38MAPK-I), cells stably transduced with SC shRNA, cells stably transduced with Twist-shRNA, cells stably transfected with GDF15, cells stably transduced with Twist-shRNA and transfected with GDF15 (Twist-shRNA + GDF15) and cells stably transfected with GDF15 and treated with SB203580 $(10 \mu \mathrm{M})$ for $30 \mathrm{~min}(\mathrm{GDF} 15+\mathrm{p} 38 \mathrm{MAPK}-\mathrm{I})$. The Twist and the GDF15 mRNA levels are shown as fold changes to those of NC (designated as 1). Each experiment was repeated three times in duplicate. Data values are expressed as the mean + standard deviation. ${ }^{\mathrm{a}} \mathrm{P}<0.05$ vs. controls (NC, VC and SC); ${ }^{\mathrm{P}}<0.05$ vs. Twist; ${ }^{\mathrm{C}} \mathrm{P}<0.05$ vs. GDF15-shRNA; ${ }^{\mathrm{d}} \mathrm{P}<0.05$ vs. Twist + GDF15-shRNA; ${ }^{\mathrm{e}} \mathrm{P}<0.05$ vs. Twist + p38MAPK-I; ${ }^{\mathrm{f}} \mathrm{P}<0.05$ vs. Twist-shRNA; ${ }^{\mathrm{P}} \mathrm{P}<0.05$ vs. GDF15. GDF15, growth differentiation factor 15 ; PC, pancreatic cancer; NC, normal control; MAPK, mitogen-activated protein kinase; SC, scrambled control.

expression in PC cells by a p38 MAPK-dependent mechanism (data not shown). Therefore, a selective p38 MAPK inhibitor SB203580 $(10 \mu \mathrm{M})$ was included in all experiments in the present study (23). As shown in Fig. 1, the p38 MAPK inhibitor had no significant effect on the constitutive expression level of Twist, whereas it eradicated Twist-induced GDF15 expression in PC cells. RT-qPCR assays revealed a similar data trend (Fig. 2), suggesting that Twist regulates GDF15 expression at the mRNA level.

Effects of overexpression and knockdown of Twist and GDF15 on PC cell invasion and MMP-2 expression/activity. To examine the individual effect of and interaction between Twist

Figure 3. Effect of overexpression and knockdown of Twist and GDF15 on the invasion of PC cells. In vitro cell invasion assays were performed in (A) ASPC-1 and (B) BXPC-3 PC cells. Cell invasion in NC cells, cells stably transfected with the empty pcDNA3.1 vector (VC), cells stably transfected with Twist, cells stably transduced with GDF15-shRNA, cells stably transfected with Twist and transduced with GDF15-shRNA (Twist + GDF15-shRNA), cells stably transfected with Twist and treated with the selective p38 MAPK inhibitor SB203580 $(10 \mu \mathrm{M})$ for $30 \mathrm{~min}$ (Twist + p38MAPK-I), cells stably transduced with SC shRNA, cells stably transduced with Twist-shRNA, cells stably transfected with GDF15, cells stably transduced with Twist-shRNA and transfected with GDF15 (Twist-shRNA + GDF15) and cells stably transfected with GDF15 and treated with SB203580 $(10 \mu \mathrm{M})$ for $30 \mathrm{~min}$ (GDF15 + p38MAPK-I) determined by fluorescence and shown as fold changes to that of $\mathrm{NC}$ (designated as 1). Each experiment was repeated three times in duplicate. Data values are expressed as the mean + standard deviation. ${ }^{\mathrm{a}} \mathrm{P}<0.05$ vs. controls (NC, VC and SC); ${ }^{\mathrm{b}} \mathrm{P}<0.05$ vs. Twist; ${ }^{\mathrm{C}} \mathrm{P}<0.05$ vs. GDF15-shRNA; ${ }^{\mathrm{d}} \mathrm{P}<0.05$ vs. Twist + GDF15-shRNA; ${ }^{\mathrm{e}} \mathrm{P}<0.05$ vs. Twist $+\mathrm{p} 38 \mathrm{MAPK}-\mathrm{I} ;{ }^{\mathrm{f}} \mathrm{P}<0.05$ vs. Twist-shRNA; ${ }^{\mathrm{g}} \mathrm{P}<0.05$ vs. GDF15. GDF15, growth differentiation factor 15; $\mathrm{PC}$, pancreatic cancer; NC, normal control; MAPK, mitogen-activated protein kinase; SC, scramble control.

and GDF15 on PC cell invasion, in vitro cell invasion assays were performed. Compared with the controls, overexpression of Twist increased cell invasion by 3.4 and 2.1 fold in ASPC- 1 and BXPC- 3 cells, respectively, which was eradicated by knockdown of GDF15 or SB203580 (10 $\mu \mathrm{M}$; Fig. 3). By contrast, knockdown of Twist decreased cell invasion by $\sim 60 \%$ in ASPC-1 and BXPC-3 cells, respectively, which was completely reversed by overexpression of GDF15 (Fig. 3). Compared with the controls, overexpression of GDF15 increased cell invasion by 3.7 fold in ASPC-1 cells and 2.3 fold in BXPC-3 cells, whereas knockdown of GDF15 decreased cell invasion by $\sim 85 \%$ in the two cell lines (Fig. 3).

MMPs have a critical role in cancer cell invasion (24). Among the different MMPs assessed, MMP-2 expression was found to be significantly altered by Twist and GDF15 

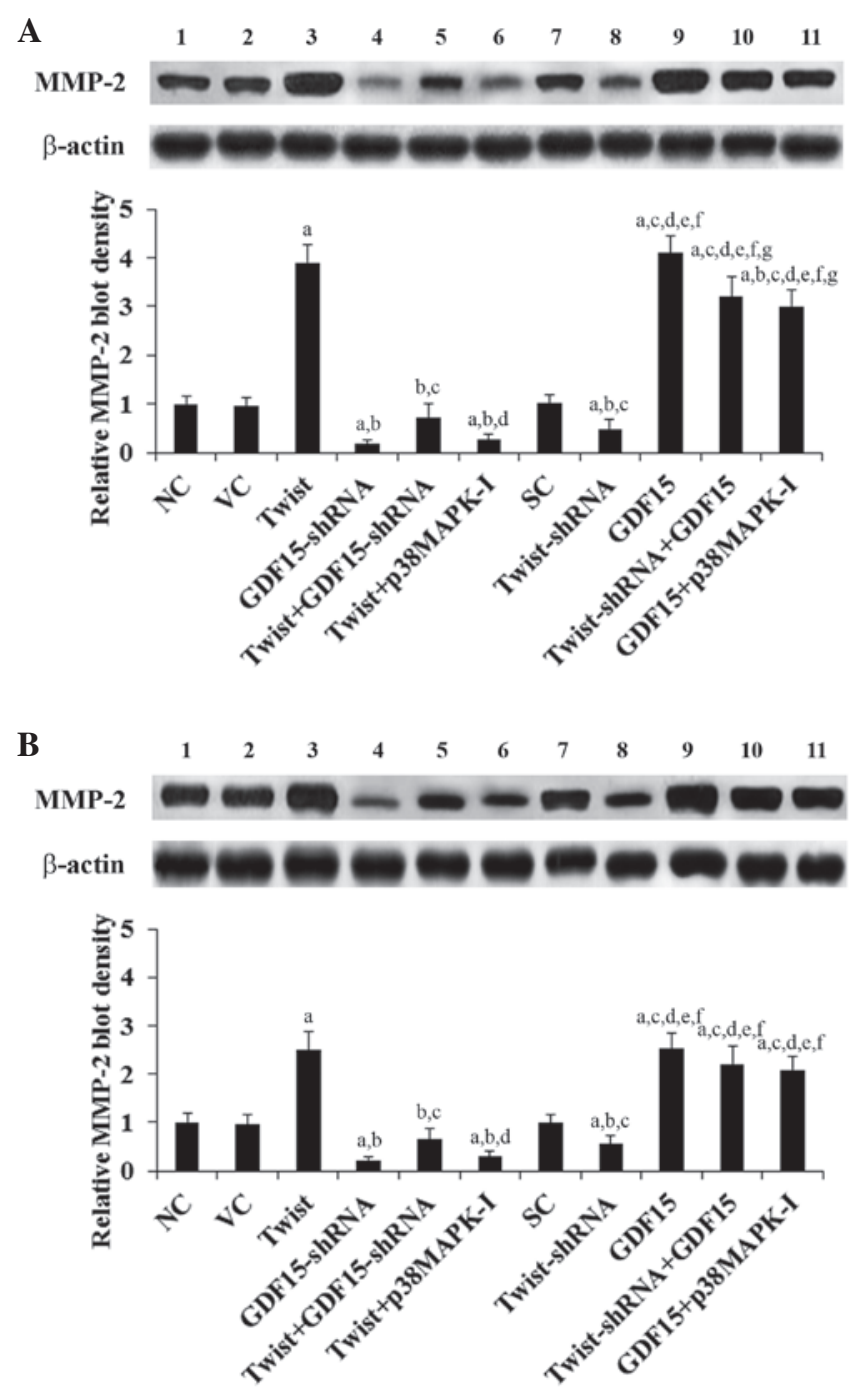

Figure 4. Effect of overexpression and knockdown of Twist and GDF15 on MMP-2 expression in PC cells. In (A) ASPC-1 and (B) BXPC-3 PC cells, the expression of MMP-2 was determined by western blot analysis in NC cells (NC, lane 1), cells stably transfected with the empty pcDNA3.1 vector (VC, lane 2), cells stably transfected with Twist (lane 3), cells stably transduced with GDF15-shRNA (lane 4), cells stably transfected with Twist and transduced with GDF15-shRNA (Twist + GDF15-shRNA, lane 5), cells stably transfected with Twist and treated with the selective p38 MAPK inhibitor SB203580 $(10 \mu \mathrm{M})$ for $30 \mathrm{~min}$ (Twist + p38MAPK-I, lane 6), cells stably transduced with SC shRNA (SC, lane 7), cells stably transduced with Twist-shRNA (lane 8), cells stably transfected with GDF15 (lane 9), cells stably transduced with Twist-shRNA and transfected with GDF15 (Twist-shRNA + GDF15, lane 10) and cells stably transfected with GDF15 and treated with SB203580 $(10 \mu \mathrm{M})$ for $30 \mathrm{~min}$ (GDF15 + p38MAPK-I, lane 11). $\beta$-actin was used as a loading control. The density of the MMP-2 blot was normalized against that of the $\beta$-actin blot to obtain a relative blo density, which is expressed as fold changes to that of NC (designated as 1). Three independent experiments were performed for each western blot analysis. Data values are expressed as the mean + standard deviation. ${ }^{\mathrm{a}} \mathrm{P}<0.05$ vs controls (NC, VC and SC); ${ }^{\mathrm{b}} \mathrm{P}<0.05$ vs. Twist; ${ }^{\mathrm{C}} \mathrm{P}<0.05$ vs. GDF15-shRNA; ${ }^{\mathrm{d}} \mathrm{P}<0.05$ vs. Twist + GDF15-shRNA; ${ }^{\mathrm{e}} \mathrm{P}<0.05$ vs. Twist + p38MAPK-I ${ }^{\mathrm{f}} \mathrm{P}<0.05$ vs. Twist-shRNA; ${ }^{\mathrm{g}} \mathrm{P}<0.05$ vs. GDF15. GDF15, growth differentiation factor 15; MMP-2, matrix metalloproteinase-2; PC, pancreatic cancer $\mathrm{NC}$, normal control; MAPK, mitogen-activated protein kinase; SC, scramble control.

in PC cells (data not shown). Compared with the controls, overexpression of Twist increased MMP-2 expression by 3.9 and 2.5 fold in ASPC-1 and BXPC-3 cells, respectively, which was eradicated by knockdown of GDF15 or SB203580
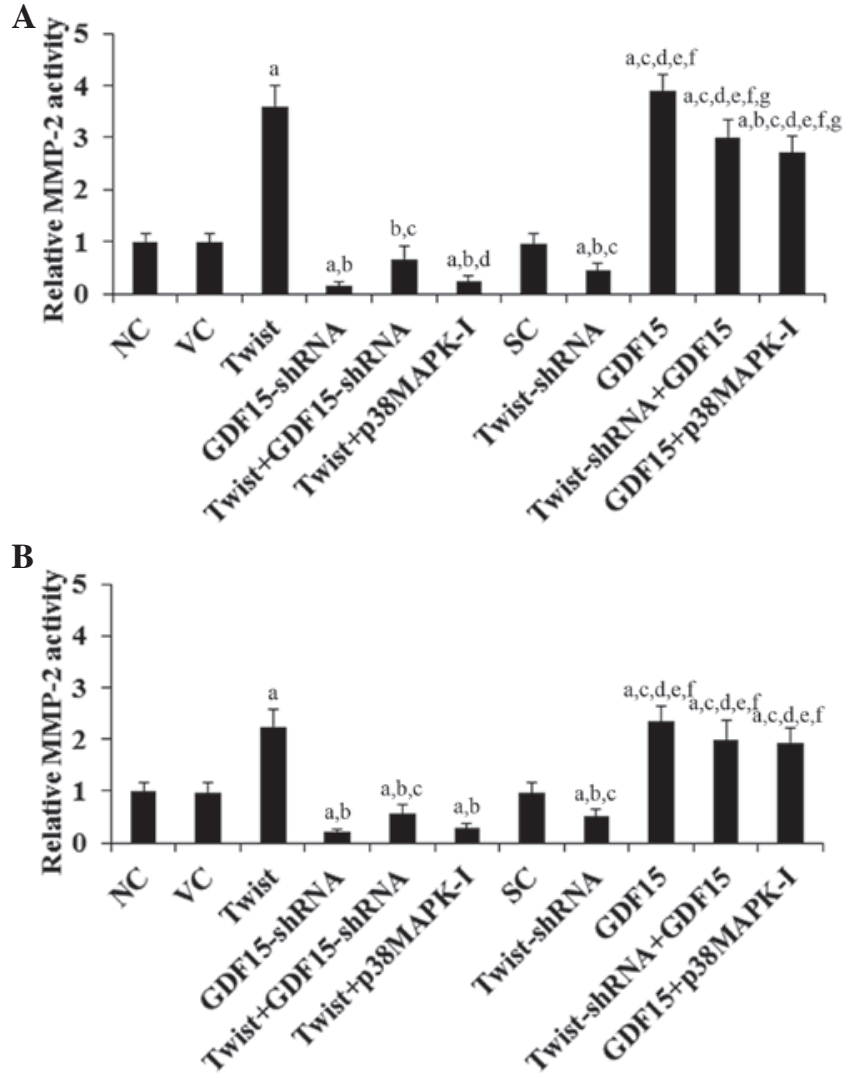

Figure 5. Effect of overexpression and knockdown of Twist and GDF15 on MMP-2 activity in PC cells. In (A) ASPC-1 and (B) BXPC-3 PC cells, the MMP-2 activity was determined in NC cells, cells stably transfected with the empty pcDNA3.1 vector (VC), cells stably transfected with Twist, cells stably transduced with GDF15-shRNA, cells stably transfected with Twist and transduced with GDF15-shRNA (Twist + GDF15-shRNA), cells stably transfected with Twist and treated with the selective p38 MAPK inhibitor SB203580 $(10 \mu \mathrm{M})$ for $30 \mathrm{~min}$ (Twist + p38MAPK-I), cells stably transduced with SC shRNA (SC), cells stably transduced with Twist-shRNA, cells stably transfected with GDF15, cells stably transduced with Twist-shRNA and transfected with GDF15 (Twist-shRNA + GDF15) and cells stably transfected with GDF15 and treated with SB203580 $(10 \mu \mathrm{M})$ for $30 \mathrm{~min}$ (GDF15 + p38MAPK-I). The MMP-2 activity is shown as fold changes to that of NC (designated as 1). Each experiment was repeated three times in duplicate. Data values are expressed as the mean + standard deviation. Each experiment was repeated three times in duplicate. Data values are expressed as the mean + standard deviation. ${ }^{\mathrm{a}} \mathrm{P}<0.05$ vs. controls $(\mathrm{NC}$, VC and SC); ${ }^{\mathrm{b}} \mathrm{P}<0.05$ vs. Twist; ${ }^{\mathrm{C}} \mathrm{P}<0.05$ vs. GDF 15 -shRNA; ${ }^{\mathrm{d}} \mathrm{P}<0.05$ vs. Twist+GDF15-shRNA; ${ }^{\mathrm{e}} \mathrm{P}<0.05$ vs. Twist+p38MAPK-I; ${ }^{\mathrm{f}} \mathrm{P}<0.05$ vs. Twist-shRNA; ${ }^{\mathrm{P}} \mathrm{P}<0.05$ vs. GDF15. GDF15, growth differentiation factor 15 ; MMP-2, matrix metalloproteinase-2; PC, pancreatic cancer; NC, normal control; MAPK, mitogen-activated protein kinase; SC, scramble control.

(10 $\mu \mathrm{M}$; Fig. 4). By contrast, knockdown of Twist decreased MMP-2 expression by $51 \%$ in ASPC-1 and $43 \%$ in BXPC-3 cells, which was completely reversed by overexpression of GDF15 (Fig. 4). Compared with the controls, overexpression of GDF15 increased MMP-2 expression by 4.1 fold in ASPC-1 cells and 2.5 fold in BXPC-3 cells, whereas knockdown of GDF15 decreased MMP-2 expression by $\sim 80 \%$ in the two cell lines (Fig. 4). A similar data trend was observed with MMP-2 activity (Fig. 5).

Effects of overexpression and knockdown of Twist and GDF15 on PC cell chemoresistance to cisplatin. To examine the individual effect of and interaction between Twist and GDF15 on 

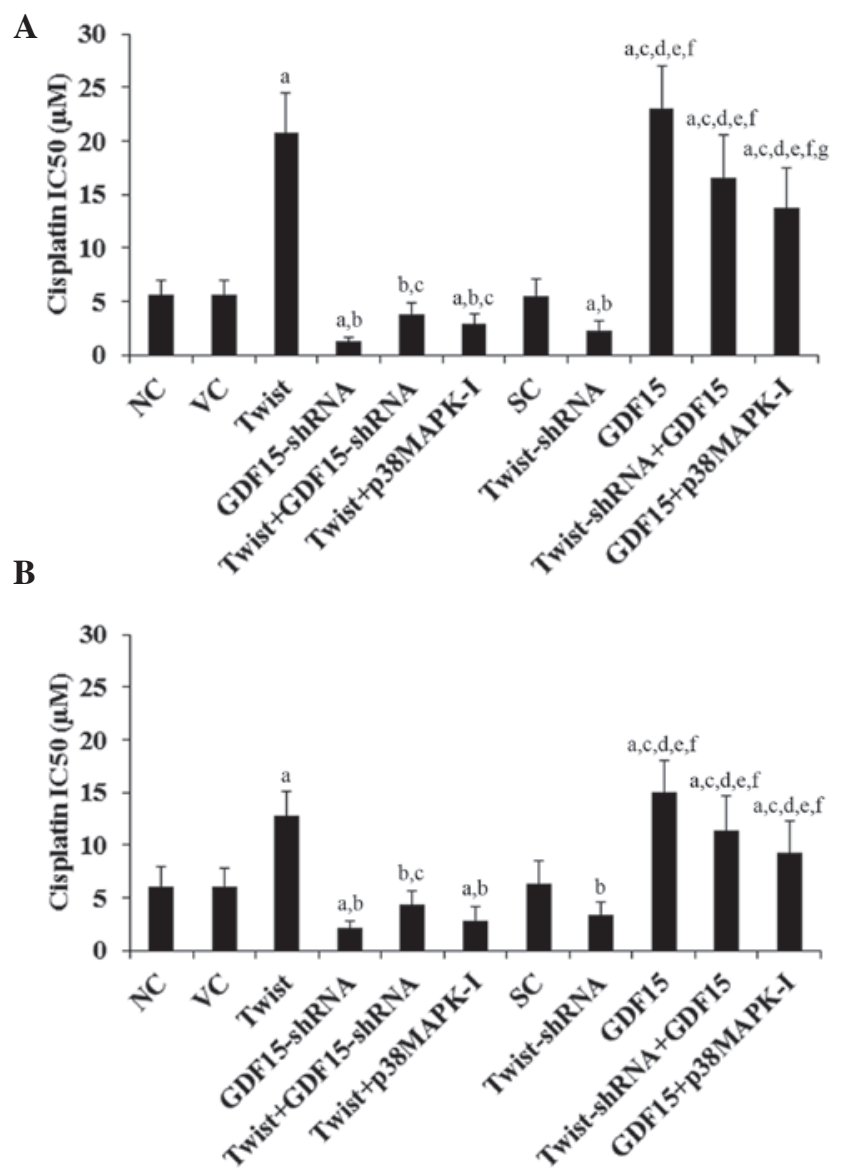

Figure 6. Effect of overexpression and knockdown of Twist and GDF15 on chemoresistance to cisplatin in PC cells. (A) ASPC-1 and (B) BXPC-3 PC cells were treated with or without various concentrations of cisplatin for $48 \mathrm{~h}$. The half maximal inhibitory concentration (IC50) values were determined in NC cells, cells stably transfected with the empty pcDNA3.1 vector (VC), cells stably transfected with Twist, cells stably transduced with GDF15-shRNA, cells stably transfected with Twist and transduced with GDF15-shRNA (Twist + GDF15-shRNA), cells stably transfected with Twist and treated with the selective p38 MAPK inhibitor SB203580 $(10 \mu \mathrm{M})$ for 30 min (Twist + p38MAPK-I), cells stably transduced with SC shRNA (SC), cells stably transduced with Twist-shRNA, cells stably transfected with GDF15, cells stably transduced with Twist-shRNA and transfected with GDF15 (Twist-shRNA + GDF15) and cells stably transfected with GDF15 and treated with SB203580 $(10 \mu \mathrm{M})$ for 30 min (GDF15 + p38MAPK-I). Each experiment was repeated three times in duplicate. Data values are expressed as the mean + standard deviation. ${ }^{a} \mathrm{P}<0.05$ vs. controls $(\mathrm{NC}$, VC and SC); ${ }^{b} \mathrm{P}<0.05$ vs. Twist; ${ }^{\circ} \mathrm{P}<0.05$ vs. GDF15-shRNA; ${ }^{\mathrm{d}} \mathrm{P}<0.05$ vs. Twist+GDF15-shRNA; ${ }^{\mathrm{e}} \mathrm{P}<0.05$ vs. Twist+p38MAPK-I; ${ }^{\mathrm{f}} \mathrm{P}<0.05$ vs. Twist-shRNA; ' $\mathrm{P}<0.05$ vs. GDF15. GDF15, growth differentiation factor 15 ; PC, pancreatic cancer; NC, normal control; MAPK, mitogen-activated protein kinase; SC, scramble control.

PC chemoresistance, cisplatin IC50 values were examined in PC cells. A higher IC50 value was considered to correspond with clinical chemoresistance to cisplatin. As shown in Fig. 6, after $48 \mathrm{~h}$ of cisplatin treatment, the cisplatin IC50 values for ASPC-1 and BXPC-3 cells were 5.6 and $6.1 \mu \mathrm{M}$, respectively. Overexpression of Twist significantly increased the IC50 values to 20.5 and $12.7 \mu \mathrm{M}$, respectively, which was eradicated by knockdown of GDF15 or SB203580 (10 $\mu$ M; Fig. 6). By contrast, knockdown of Twist decreased the IC50 values to 2.3 and $3.4 \mu \mathrm{M}$, which was completely reversed by overexpression of GDF15 (Fig. 6). Overexpression of GDF15 increased the IC50 values of ASPC- 1 and BXPC- 3 cells to 23.2 and

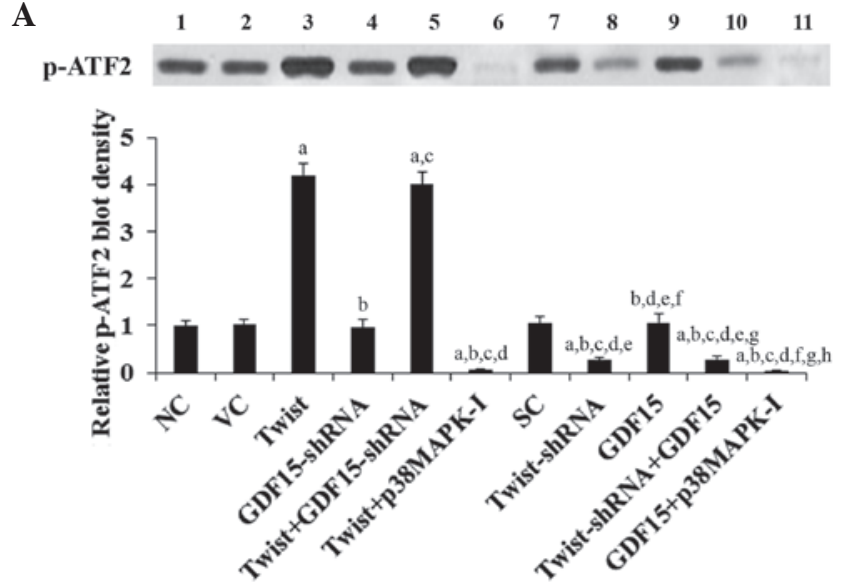

B
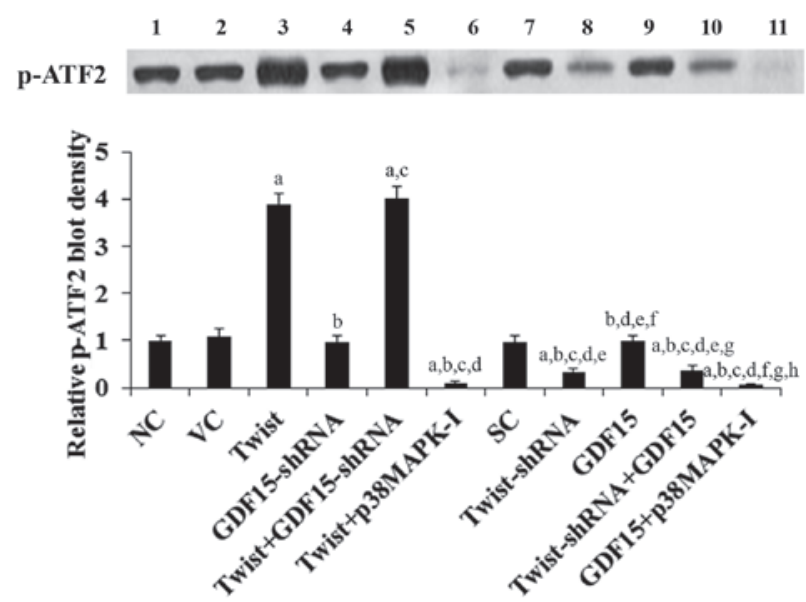

Figure 7. Effect of overexpression and knockdown of Twist and GDF15 on p38 MAPK activity in PC cells. In (A) ASPC-1 and (B) BXPC-3 PC cells, the $\mathrm{p} 38$ MAPK activity was determined by measuring the phosphorylation of ATF2, a substrate of activated p38 MAPK. The levels of p-ATF2 were determined by western blot analysis in $\mathrm{NC}$ cells $(\mathrm{NC}$, lane 1), cells stably transfected with the empty pcDNA3.1 vector (VC, lane 2), cells stably transfected with Twist (lane 3), cells stably transduced with GDF15-shRNA (lane 4), cells stably transfected with Twist and transduced with GDF15-shRNA (Twist + GDF15-shRNA, lane 5), cells stably transfected with Twist and treated with the selective p38 MAPK inhibitor SB203580 $(10 \mu \mathrm{M})$ for $30 \mathrm{~min}$ (Twist + p38MAPK-I, lane 6), cells stably transduced with SC shRNA (SC, lane 7), cells stably transduced with Twist-shRNA (lane 8), cells stably transfected with GDF15 (lane 9), cells stably transduced with Twist-shRNA and transfected with GDF15 (Twist-shRNA + GDF15, lane 10) and cells stably transfected with GDF15 and treated with SB203580 $(10 \mu \mathrm{M})$ for $30 \mathrm{~min}$ (GDF15 + p38MAPK-I, lane 11). The p38 MAPK activity is shown as fold changes to that of $\mathrm{NC}$ (designated as 1). Each experiment was repeated three times in duplicate. Data are expressed as the mean + standard deviation. ${ }^{\mathrm{a}} \mathrm{P}<0.05$ vs. controls $\left(\mathrm{NC}, \mathrm{VC}\right.$ and $\mathrm{SC}$ ); ${ }^{\mathrm{b}} \mathrm{P}<0.05$ vs. Twist; ${ }^{\mathrm{c}} \mathrm{P}<0.05$ vs. GDF15-shRNA; ${ }^{\mathrm{d}} \mathrm{P}<0.05$ vs. Twist $+\mathrm{GDF} 15$-shRNA; ${ }^{\mathrm{P}} \mathrm{P}<0.05$ vs. Twist + p38MAPK-I; ${ }^{\mathrm{f}} \mathrm{P}<0.05$ vs. Twist-shRNA; ${ }^{\mathrm{g}} \mathrm{P}<0.05$ vs. GDF15; ${ }^{\text {h}} \mathrm{P}<0.05$ vs. Twist-shRNA + GDF15. GDF15, growth differentiation factor 15; MAPK, mitogen-activated protein kinase; PC, pancreatic cancer; NC, normal control; MAPK, mitogen-activated protein kinase; ATF2, activating transcription factor 2; p-ATF2, phosphorylated ATF2; SC, scramble control.

$15.4 \mu \mathrm{M}$, respectively, while knockdown of GDF15 decreased the IC50 values to 1.3 and $2.1 \mu \mathrm{M}$, respectively (Fig. 6).

Effects of overexpression and knockdown of Twist and GDF15 on $p 38$ MAPK activity in PC cells. The above results suggested that Twist promotes PC cell invasion and chemoresistance to cisplatin largely through regulating GDF15 expression by a 38 
MAPK-dependent mechanism. Therefore, the individual effect of and interaction between Twist and GDF15 on p38 MAPK activity was next examined, which was measured by phosphorylation of ATF2, a substrate of activated p38 MAPK (22). As evidenced by increased levels of phosphorylated ATF2, overexpression of Twist induced p38 MAPK activity by 4.2 and 3.9 fold in ASPC-1 and BXPC-3 cells, respectively, which was eradicated by SB203580 $(10 \mu \mathrm{M})$ but not knockdown of GDF15 (Fig. 7). By contrast, knockdown of Twist decreased p38 MAPK activity by $70 \%$ in ASPC-1 and BXPC-3 cells, which was not significantly affected by overexpression of GDF15 (Fig. 7). Compared with the controls, overexpression and knockdown of GDF15 demonstrated no significant effect on p38 MAPK activity (Fig. 7).

\section{Discussion}

The present study demonstrated that Twist promotes PC cell invasion and cisplatin chemoresistance largely through GDF15. Overexpression and knockdown of Twist in PC cells increased and decreased the expression of GDF15, respectively, at the mRNA and the protein levels, but not vice versa. The findings suggest that Twist induces GDF15 expression in PC cells at the gene transcription/mRNA level. In addition, a selective p38 MAPK inhibitor readily eliminated Twist-induced GDF15 expression in PC cells without significantly altering the expression of Twist, indicating that Twist induces GDF15 expression in a p38 MPAK-dependent manner in PC cells. How Twist transcriptionally regulates the expression of GDF15 through p38 MAPK in PC cells will be examined in future studies.

As evidenced by gene overexpression and knockdown experiments, Twist and GDF15 individually promotes PC cell invasion and cisplatin resistance. In addition, knockdown of GDF15 eradicated the stimulatory effects of overexpressing Twist, while overexpression of GDF15 completely reversed the inhibitory effects of knocking down Twist. The findings indicate that GDF15 is functionally downstream of Twist and largely mediates the promoting effects of Twist on PC cell invasion and cisplatin resistance, which corroborates our finding that Twist induces GDF15 expression in PC cells.

While the selective p38 MAPK inhibitor SB203580 abrogated the promoting effects of Twist overexpression on PC cell invasion and cisplatin resistance, overexpression of GDF15 significantly augmented PC cell invasion and cisplatin resistance in the presence of SB203580. The results suggest that Twist and GDF15 act functionally upstream and downstream of p38 MAPK, respectively. This is in agreement with our findings that while overexpression and knockdown of Twist increased and decreased p38 MAPK activity, respectively, GDF15 demonstrated no significant effect on p38 MAPK activity in PC cells. Previous studies have suggested an important role of p38 MAPK in PC cell invasion $(25,26)$. Our findings indicate that p38 MAPK mediates Twist-induced GDF15 expression in PC cells, which markedly promotes PC cell invasion. Thus, the importance of p38 MAPK signaling in PC progression is at least partially fulfilled through Twist/GDF15 signaling.

MMPs are critical for cancer cell invasion $(17,18)$. Previous studies have suggested that MMP-2 is important for PC cell invasion in vitro (26). In the present study, it was found that Twist markedly increased MMP-2 expression/activity through
GDF15, suggesting that the Twist/GDF15 signaling axis is important for PC progression. GDF15 has been reported to have tumorigenic and anti-tumorigenic activities $(11,12)$. While Twist has been widely associated with the initial phase of metastatic progression (8), a previous study demonstrated that Twist decreases cisplatin resistance in osteosarcoma cells, suggesting that Twist, like GDF15, has a dual role in cancer cell malignancy and chemoresistance, depending on tissue specificity (27). Since Twist and GDF15 have been found to be overexpressed in various types of cancer $(8,28)$, it would be of significance to define the role of the Twist/GDF15 signaling axis in other types of cancer besides PC in future studies.

In conclusion, the present study for the first time, to the best of our knowledge, demonstrated that Twist promotes PC cell invasion and cisplatin chemoresistance through inducing GDF15 expression by a p38 MAPK-dependent mechanism. This adds new insights into the molecular mechanisms underlying PC progression and chemoresistance.

\section{References}

1. Jiang H, Duan B, He C, et al: Cytoplasmic HSP90 $\alpha$ expression is associated with perineural invasion in pancreatic cancer. Int J Clin Exp Pathol 7: 3305-3311, 2014.

2. Spano JP, Chodkiewicz C, Maurel J, et al: Efficacy of gemcitabine plus axitinib compared with gemcitabine alone in patients with advanced pancreatic cancer: An open-label randomised phase II study. Lancet 371: 2101-2108, 2008.

3. López R, Méndez CM,Fernández MJ, et al: Phase II trial of erlotinib plus capecitabine as first-line treatment for metastatic pancreatic cancer (XELTA study). Anticancer Res 33: 717-723, 2013.

4. Hidalgo M: Pancreatic cancer. N Engl J Med 362: 1605-1617, 2010.

5. Chung TW, Choi HJ, Kim SJ, et al: The ganglioside GM3 is associated with cisplatin-induced apoptosis in human colon cancer cells. PLoS One 9: e92786, 2014.

6. Gresham GK, Wells GA, Gill S, Cameron C and Jonker DJ: Chemotherapy regimens for advanced pancreatic cancer: A systematic review and network meta-analysis. BMC Cancer 14: 471,2014

7. Ramfidis VS, Psyrri A, Syrigos KN and Saif MW: First line treatment for metastatic pancreatic adenocarcinoma: Looking for the step forward. JOP 15: 286-288, 2014.

8. Entz-Werlé N, Choquet P, Neuville A, et al: Targeted apc;twist double-mutant mice: A new model of spontaneous osteosarcoma that mimics the human disease. Transl Oncol 3: 344-353, 2010.

9. Ohuchida K, Mizumoto K, Ohhashi S, et al: Twist, a novel oncogene, is upregulated in pancreatic cancer: Clinical implication of Twist expression in pancreatic juice. Int J Cancer 120: 1634-1640, 2007.

10. Breit SN, Johnen H, Cook AD, et al: The TGF- $\beta$ superfamily cytokine, MIC-1/GDF15: A pleotrophic cytokine with roles in inflammation, cancer and metabolism. Growth Factors 29: 187-195, 2011.

11. Mimeault M and Batra SK: Divergent molecular mechanisms underlying the pleiotropic functions of macrophage inhibitory cytokine-1 in cancer. J Cell Physiol 224: 626-635, 2010.

12. Khaled YS, Elkord E and Ammori BJ: Macrophage inhibitory cytokine-1: A review of its pleiotropic actions in cancer. Cancer Biomark 11: 183-190, 2012.

13. Koopmann J, Buckhaults P, Brown DA, et al: Serum macrophage inhibitory cytokine 1 as a marker of pancreatic and other periampullary cancers. Clin Cancer Res 10: 2386-2392, 2004.

14. Ozkan H, Demirbaş S, Ibiş M, Akbal E and Köklü S: Diagnostic validity of serum macrophage inhibitor cytokine and tissue polypeptide-specific antigen in pancreatobiliary diseases. Pancreatology 11: 295-300, 2011.

15. Chen YZ, Liu D, Zhao YX, Wang HT, Gao Y and Chen Y: Diagnostic performance of serum macrophage inhibitory cytokine-1 in pancreatic cancer: A meta-analysis and meta-regression analysis. DNA Cell Biol 33: 370-377, 2014.

16. Matsuo N, Shiraha H, Fujik T, et al: Twist expression promotes migration and invasion in hepatocellular carcinoma. BMC Cancer 9: 240, 2009. 
17. Wang B, Feng P, Xiao Z and Ren EC: LIM and SH3 protein 1 (Lasp1) is a novel p53 transcriptional target involved in hepatocellular carcinoma. J Hepatol 50: 528-537, 2009.

18. Feng Y, Hu J, Ma J, et al: RNAi-mediated silencing of VEGF-C inhibits non-small cell lung cancer progression by simultaneously down-regulating the CXCR4, CCR7, VEGFR-2 and VEGFR-3-dependent axes-induced ERK, p38 and AKT signalling pathways. Eur J Cancer 47: 2353-2363, 2011.

19. Jo YK, Park SJ, Shin JH, et al: ARP101, a selective MMP-2 inhibitor, induces autophagy-associated cell death in cancer cells. Biochem Biophys Res Commun 404: 1039-1043, 2011.

20. Qazi H, Shi ZD and Tarbell JM: Fluid shear stress regulates the invasive potential of glioma cells via modulation of migratory activity and matrix metalloproteinase expression. PLoS One 6 : e20348, 2011.

21. Ding X,Zhang Z, Li S and Wang A: Combretastatin A4 phosphate induces programmed cell death in vascular endothelial cells. Oncol Res 19: 303-309, 2011.

22. Wang $\mathrm{X}, \mathrm{Wu} \mathrm{H}$ and Miller AH: Interleukin 1alpha (IL-1alpha) induced activation of p38 mitogen-activated protein kinase inhibits glucocorticoid receptor function. Mol Psychiatry 9: 65-75, 2004.
23. Leelahavanichkul K, Amornphimoltham P, Molinolo AA, Basile JR, Koontongkaew S and Gutkind JS: A role for p38 MAPK in head and neck cancer cell growth and tumor-induced angiogenesis and lymphangiogenesis. Mol Oncol 8: 105-118, 2014.

24. Li Y, Liao Q, Li K, Zhong D, Weng X and Mi M: Knockdown of endothelin A receptor expression inhibits osteosarcoma pulmonary metastasis in an orthotopic xenograft mouse model. Mol Med Rep 5: 1391-1395, 2012.

25. Cui XP, Qin CK, Zhang ZH, et al: HOXA10 promotes cell invasion and MMP-3 expression via TGF 32 -mediated activation of the p38 MAPK pathway in pancreatic cancer cells. Dig Dis Sci 59: 1442-1451, 2014.

26. Pan F, Ma S, Cao W, et al: SDF-1 $\alpha$ upregulation of MMP-2 is mediated by p38 MAPK signaling in pancreatic cancer cell lines. Mol Biol Rep 40: 4139-4146, 2013.

27. Wu J, Liao Q, He H, Zhong D and Yin K: TWIST interacts with $\beta$-catenin signaling on osteosarcoma cell survival against cisplatin. Mol Carcinog 53: 440-446, 2014.

28. Bauskin AR, Brown DA, Kuffner T, et al: Role of macrophage inhibitory cytokine-1 in tumorigenesis and diagnosis of cancer. Cancer Res 66: 4983-4986, 2006. 